1895. Mille huit cent quatre-vingt-quinze

Revue de l'association française de recherche sur

I'histoire du cinéma

$61 \mid 2010$

Aux sources du burlesque cinématographique : les comiques français des premiers temps

\title{
Les galipettes de l'Autre burlesque ou la mécanique corporelle du Double
}

Rae Beth Gordon

\section{OpenEdition}

Journals

Édition électronique

URL : https://journals.openedition.org/1895/3834

DOI : 10.4000/1895.3834

ISBN : 978-2-8218-0980-2

ISSN : $1960-6176$

Éditeur

Association française de recherche sur l'histoire du cinéma (AFRHC)

Édition imprimée

Date de publication : 1 septembre 2010

Pagination : 128-148

ISBN : 978-2-913758-62-9

ISSN : 0769-0959

\section{Référence électronique}

Rae Beth Gordon, «Les galipettes de l'Autre burlesque ou la mécanique corporelle du Double », 1895. Mille huit cent quatre-vingt-quinze [En ligne], 61 | 2010, mis en ligne le 01 septembre 2013, consulté le 16 avril 2022. URL : http://journals.openedition.org/1895/3834 ; DOI : https://doi.org/

$10.4000 / 1895.3834$ 
$1895 /$

$n^{\circ} 61$

septembre

2010

128

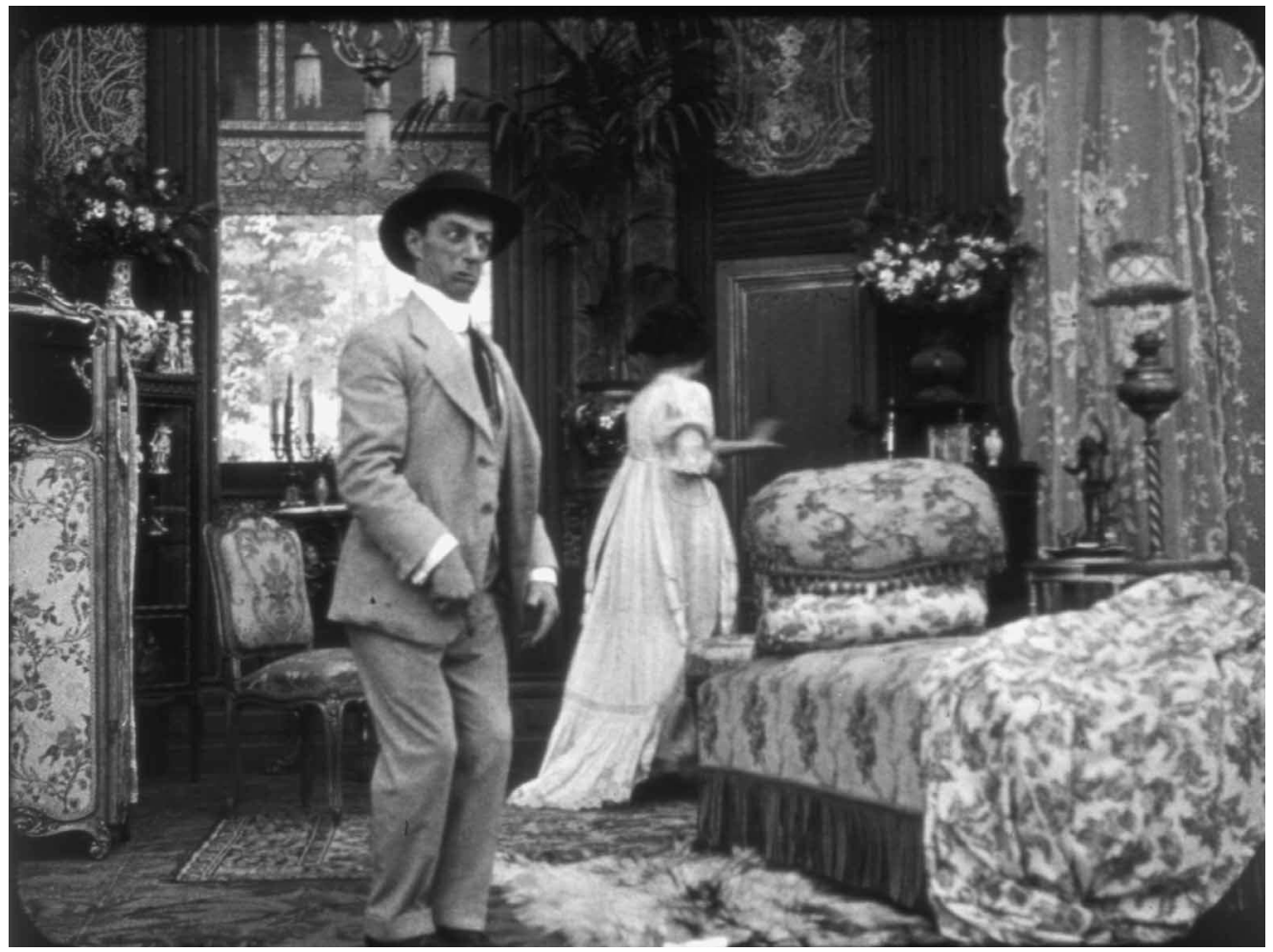

Fig. 1. Boireau et la demi-mondaine, Pathé, 1912. 


\section{Les galipettes de l'Autre burlesque ou la mécanique corporelle du Double}

par Rae Beth Gordon

La gestuelle des dégénérés

Dans les années d'émergence du cinéma, l'une des principales constructions médicales pour conceptualiser le fonctionnement de la psyché consiste en une division entre les facultés supérieures - la raison, le jugement, la volonté -, et les facultés inférieures - les fonctions sensorimotrices, les automatismes comprenant les réflexes nerveux et l'instinct. Ce sont ces facultés inférieures qui composent "l'inconscient corporel "1. Cette conception d'une division primordiale entre supérieur et inférieur est élargie pour inclure les théories de Charles Darwin et de Herbert Spencer sur l'évolution, impliquant la notion d'une potentielle régression ou d'un retour vers l'animalité. En effet, l'idée d'une évolution à rebours va être de plus en plus intégrée dans les études médicales sur la dégénérescence. On classe sous la rubrique « dégénéré » les hystériques, les épileptiques, les neurasthéniques, les pervers, les alcooliques et les idiots, tous sous la domination des facultés inférieures². Le style et les thèmes du cinéma burlesque en France, surtout jusqu'en 1913, font ouvertement appel aux facultés inférieures et à la régression. La culture de masse - café-concert, foire, cinéma - était d'ailleurs vue à la lumière des théories psychologiques et anthropologiques, cette « culture du bas » étant associée au goût des dégénérés pour les spectacles affichant un univers de régression. Grâce à la presse populaire, les symptômes effrayants et sensationnels des maladies nerveuses frappaient l'imagination, et c'est pourquoi le public du cinéma reconnaissait leurs caractéristiques dans les films burlesques, voyant l'éruption de l'inconscient corporel dans les mouvements mêmes des acteurs. Cet essai propose, dans le contexte des notions médicales et psycho-physiologistes de l'époque, une lecture du cinéma burlesque qui veut cerner davantage l'impact que ces films ont pu avoir sur le spectateur.

1 Le concept d'inconscient corporel prend racine en France à partir des années 1870 .

2 Entre 1905 et 1908, I'augmentation des cas de ces afflictions était considérée comme un fléau national. 
$1895 /$

Comment ce schisme inférieur/supérieur à l'intérieur de l'être s'exprime-t-il dans les films burlesques ? La manie des têtes détachées dans les films de Georges Méliès est intéressante dans ce contexte car c'est justement lorsque la raison se sépare du corps que se produisent des phénomènes automatiques et hystériques ${ }^{3}$. Pour mieux comprendre le rapport que j'entends développer entre le film burlesque et les facultés inférieures, il faut d'abord situer ces films dans la série culturelle du spectacle populaire qui comprend notamment le café-concert. Les analogies entre les mouvements convulsifs, spasmodiques et saccadés des hystériques et épileptiques de la Salpêtrière et ceux des comiques et chanteurs de café-concert de la même époque sont multiples ${ }^{4}$. Les nouveaux genres d'artistes qui ont vu le jour dans les années 1870-1890 sont le Comique et la Chanteuse Épileptiques, le Chanteur Agité, et le Comique Idiot $^{5}$. La référence aux modèles médicaux est souvent explicite dans les chansons et dans le discours journalistique qui les accompagnent. Si la gestuelle épileptique est celle qui frappait en premier lieu le spectateur, il ne faut pas ignorer que les mouvements automatiques en général étaient associés aux maladies nerveuses. En effet, le Dr Pierre Janet a écrit que les hystériques se plaignaient constamment d'avoir l'impression d'être des automates, utilisant continuellement les mots machine, automate, mécanique ${ }^{6}$. Un grand nombre d'acteurs dans les films comiques viennent du café-concert et du music-hall, et c'est pourquoi les codes et les « messages » contenus dans la gestuelle (on lit les tics, les mouvements mécaniques et épileptiques en tant que pathologie nerveuse) sont donc transmis comme auparavant au spectateur. Il y a également continuité dans les thèmes de la difformité, de la scatologie et de l'animalité en même temps que dans les rythmes des spectacles de caf'conc'.

Le titre de certains films fait un clin d'œil à la relation privilégiée entre la pathologie nerveuse et le style caf'conc' au cinéma, par exemple les Toqués de l'omnibus (les Échappés de Charenton) de Georges Méliès (1901), le Matelas Épileptique d'Alice Guy (1906), le Tic d'Étienne Arnaud (1908), Un Tic gênant de Camille de Morlhon (1908), ou le Retapeur de cervelles

3 La distinction entre la notion de l'inconscient en psychanalyse et celle, courante à la fin du XIXe siècle, est grande : celui-ci est situé dans le corps. Centrée sur l'inconscient corporel et la dissémination dans le grand public du savoir médical, mon analyse diffère ainsi d'autres lectures du burlesque, par exemple celles, très fines, de Petr Kral, ou plus récemment, l'étude extrêmement riche des figures du burlesque d'Emmanuel Dreux (le Cinéma burlesque ou la subversion par le geste, Paris, L'Harmattan, 2007), introduite par une contextualisation historique.

4 Elles sont analysées en détail dans Rae Beth Gordon, Why the French love Jerry Lewis : From Cabaret to Early Cinema (Stanford University Press, 2001). Le texte présent est tiré en partie de ce livre, notamment l'analyse des films d'Émile Cohl, Louis Feuillade, et Georges Méliès, ainsi que certaines conceptions de base en psycho-pathologie et en psycho-physiologie.

5 Parmi les plus connus dans chacun de ces genres : Darius M. et Sinöel, Émilie Bécat et Polaire, Paulus et Ouvrard père, Libert et Dranem.

6 Janet est le premier à avoir construit une théorie cohérente de l'inconscient (qu'il appelle le subconscient). Voir son magistral Automatisme psychologique : Essai sur les formes inférieures de l'activité humaine, Paris, Alcan, 1889. 
d'Émile Cohl (1910). Le Tic (Gaumont) propose un tic qui est littéralement un clin d'œil... en l'occurrence, celui d'une jeune mariée se promenant avec son mari. Ce tic provocateur attire une dizaine d'admirateurs qui la suivent, ce qui ne va pas tarder à se transformer en coursepoursuite. Une course-poursuite dont la singularité est le fait que tous, sauf un des poursuivants, exhibent une pathologie de la marche! On imagine le plaisir qu'ont eu les acteurs à rivaliser pour inventer la démarche excentrique la plus drôle ${ }^{7}$. En effet, les tics et les démarches bizarres (reconnaissables par le spectateur d'alors comme pathologiques) deviendront la signature de presque tous les comiques dans les films des trois premières décennies. Chaplin donne I'exemple le plus évident, mais André Deed n'a rien à lui envier. Jacques Tati appartient également à cette tradition. En 1929, Jean Mauclair (le fondateur du Studio 28) définit un grand nombre de films du tournant du siècle comme « le spectacle de la rigolade automatique et de drames sentimentalo-épileptiques ${ }^{9}$. L'expression controversée de cinéma primitif prend un autre sens : celui d'un cinéma qui exalte le mouvement hyperbolique, les secousses, les automatismes des pathologies nerveuses, et l'instinct - le tout accompagné des notions d'évolution régressive qui leur sont rattachées à la fin du XIXe siècle. Le versant d'animalité si marqué dans le burlesque est lui aussi lié au mouvement hyperbolique : "Plus on descend l'échelle évolutionnaire, plus les fonctions motrices sont prononcées " 10 .

Selon David Robinson, Max Linder a rompu avec une tradition de comiques en France, " pour la plupart des créatures sauvages, non-humaines, des personnages de bande dessinée "11 : Linder « avait le don du naturel et se rendait compte que les films n'avaient pas besoin d'être frénétiques du début jusqu'à la fin [...] alors que ses contemporains misaient sur leur physique grotesque ". C'est précisément mon argument ici : I'engouement pour Linder marque déjà le début de la réfutation de ce miroir explicite et cru de l'inconscient corporel dans toute sa splendeur barbare : sauvage, instinctuel, primitif, " non-humain » (c'est-à-dire mécanique, automatique). L'impression de geste automatique et saccadé est, bien sûr, rehaussée par la trépidation de l'image, telle que l'ont commentée Guistino Ferri en 1906, puis René Doumic en 1913, parmi bien d'autres ${ }^{12}$. Ces figures à peine humaines semblent être possédées, mues

7 Gilles de la Tourette en 1886 et Ernest Brissaud en 1895 ont publié les études sur ce problème neurologique, augmenté de schémas diagrammatiques, parmi lesquelles la marche spasmodique, la marche sautillante, la marche zigzaguante, etc.

8 Cet acteur est né Henri André Augustin Chapais. Voir Jean Gili, André Deed, Genova, Le Mani, 2005 pour une étude approfondie de sa carrière et de nombreux comptes rendus de ses films.

9 Jean Mauclair, I'Ami du peuple du soir, 20 décembre 1929.

10 Boris Sidis, "Doctrine of Sensory Elements », Psychological Review, vol. 15, 1908, p. 151.

11 David Robinson, "Rise and Fall of the Clowns », Sight and Sound, vol. 56, n³, 1977, p. 199.

12 La vitesse des mouvements peut aussi évoquer en 1907 " des poupées actionnées par un mécanisme d'horlogerie ", texte de 1907 d'A. Gunsberg, repris dans le Cinéma : Naissance d'un art, 1895-1920, textes choisis et présentés par Daniel Banda et José Moure, Paris, Flammarion, 2008, p. 108. 


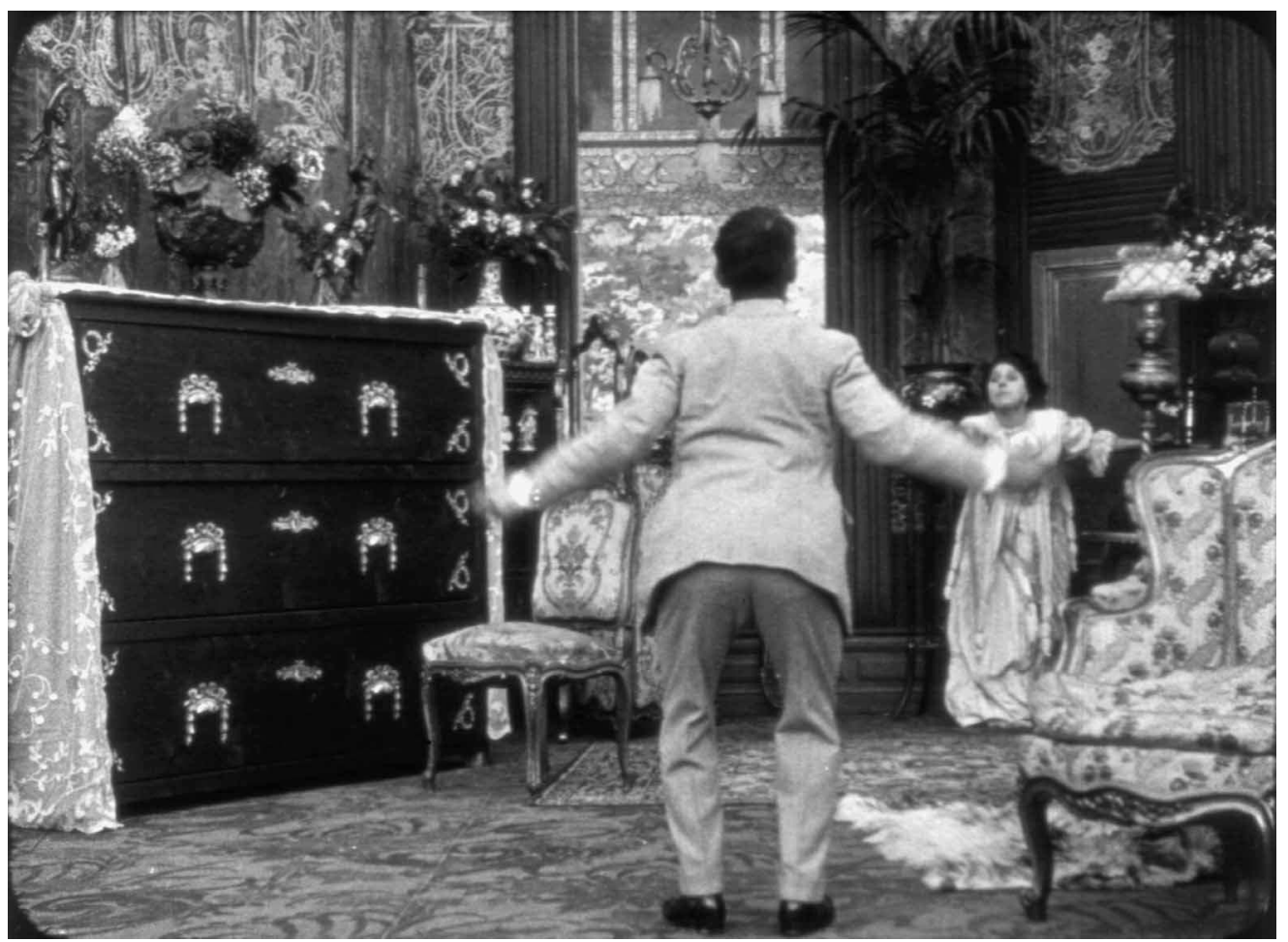

Fig. 2. Boireau et la demi-mondaine, Pathé, 1912.

par des forces démoniaques et invisibles. La force qui les agite vient des facultés inférieures : ce que certains voient comme " déshumanisé " correspond bien au registre de l'inconscient corporel. James Sully remarque ainsi que « l'effet expansif du nouveau et de l'étrange sur notre sensibilité provoque le rire ${ }^{13}{ }^{13}$. Ce même public du début du siècle riait devant les groupes d'Africains vus pour la première fois dans les expositions ethnologiques, expositions qui étaient utilisées comme confirmation des théories de régression. II est fort à parier qu'il regardait Boireau de la même façon, comme un homme $d^{\prime}$ une tribu " primitive ${ }^{14}$. Le jeu corporel d'André Deed exalte une manière d'être si autre qu'il échappe à la catégorie $\mathrm{d}^{\prime}$ homme civilisé et parfois complètement à celle de l'humain ${ }^{15}$.

13 James Sully, Essai sur le rire, Paris, Alcan, 1904.

14 Ricciotto Canudo, d'ailleurs, met en rapport le côté instructif des phénomènes de foire avec les films des « hommes les plus inconnus, des expressions humaines les plus ignorées [...] s'agitant [...] devant les regards du spectateur entrainé dans la rapidité de la figuration ", "Triomphe du Cinématographe " (1908), dans Daniel Banda et José Moure, op. cit., p. 190.

15 Thomas Brandlmeier affirme avec raison que Deed « figure comme le personnage le plus cru, primitif, des premières stars comiques », ("Fin-de-siècle Comedy Culture », dans Slapstick \& Co., H. Berlach et W. Jacobsen (dir.), Berlin, Argon, 1995, p. 35). 


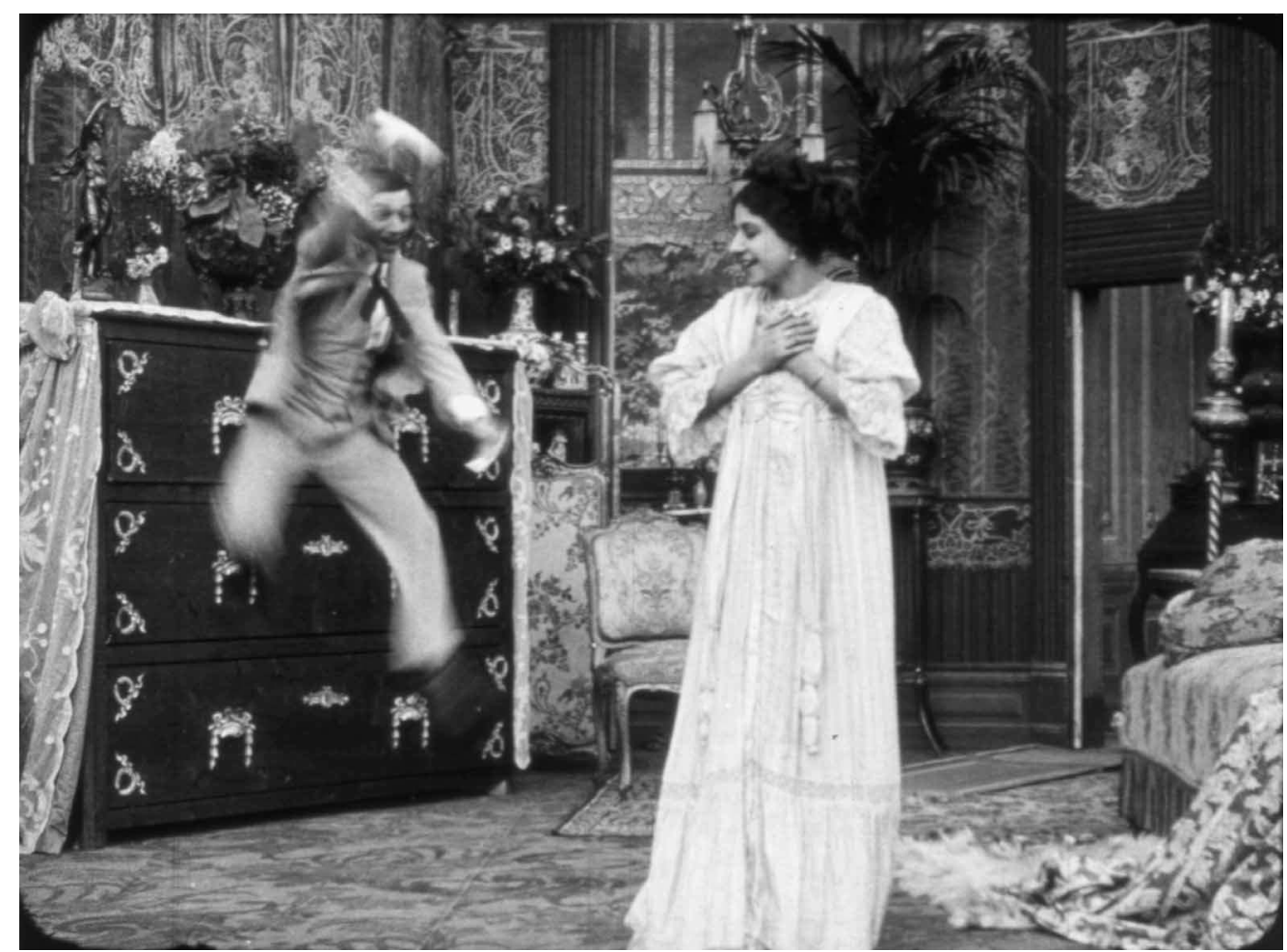

Fig. 3. Boireau et la demi-mondaine, Pathé, 1912.
1895 / $\mathrm{n}^{\circ} 61$ septembre 2010

133

Prenons le burlesque corporel particulier de Deed qui vient de la position habituelle du corps, dont la silhouette est immédiatement reconnaissable, et de l'élasticité de ce petit corps au long visage. Deed est le plus souvent dans une posture accroupie, la taille cambrée, genoux pliés et fesses en l'air (fig. 1 et 2 : Boireau et la demi-mondaine avec Valentina Frascaroli, Pathé, 1912). Sa grimace étire le bas du visage de façon grotesque, alors que sa petite taille et son attitude corporelle lui permettent de faire un effet d'agrandissement surnaturel lorsqu'il s'étire subitement vers le haut. Comme, par exemple, dans Boireau spadassin (Pathé 1913) où, à cette extension verticale, s'ajoute le bras levé surmonté d'une épée. Ou bien lorsqu'il saute subitement en l'air à une hauteur digne de Nijinsky (fig. 3). Ces surgissements évoquent on ne peut mieux un diable à ressort, parfait symbole de ce qui surgit de façon inattendue, produisant un rire d'étonnement ${ }^{16}$. Une autre forme de burlesque corporel chez

16 L'image du diable à ressort figure aussi dans la Lanterne magique de Méliès (1903). Henri Bergson donne ce jouet comme exemple de mouvement automatique, inlassablement répété, (le Rire : Essai sur la signification du comique, Paris, P.U.F., 2007 [1899], pp. 53-59).

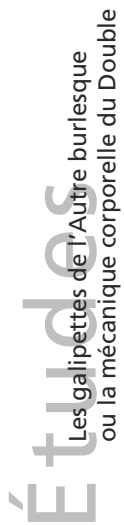


$1895 /$

Plus vite, toujours plus vite

Tel le choc perceptuel des trains dévorant l'espace, qui constituait le sujet d'un bon nombre de films dans la première décennie du cinéma, le mouvement hystérique offrait au spectateur une expérience de secousse perceptuelle et viscérale 20 . Le mouvement hystérique, comme le mouvement des trains, fait partie du paradigme essentiel de la modernité - la vitesse - dont le cinéma est le couronnement. Nous verrons bientôt que ce genre de mouvement et de gestuelle constitue un fond capital pour les films burlesques en France. Très tôt, les commentateurs considèrent le mouvement et la vélocité comme l'essence du cinéma. Canudo écrit ainsi en 1908 que "le spectacle n'existe que par excès de mouvement ", mais que « plus que le mouvement des images [...], ce qui est vraiment symbolique de la vitesse moderne, c'est le Geste des personnages »²1. Quant à René Doumic, il estime en 1913 que « le mouvement, c'est son triomphe et son essence ". Pour lui, le cinéma doit être " un art d'utiliser le mouvement pour en tirer des effets de surprise, de terreur ou de drôlerie. [...] Le parfait cinédrame, si on nous le donne quelque jour, réalisera le mouvement perpétuel ${ }^{22}$. Ce mouvement exacerbé des corps à l'intérieur du cadre filmique a son miroir dans l'activité

17 II écrira, réalisera, et jouera l'Homme mécanique (L'uomo meccanico) en 1921 en Italie. La description publicitaire de la « force terrifiante et vélocité incalculable » du personnage est intéressante dans le contexte de l'inconscient corporel. Voir Jean A. Gili, op. cit., p. 145.

18 Robert Desnos, les Rayons et les ombres, cinéma, Paris, Gallimard, 1992, p. 47.

19 Nicole Vedrès, Images du cinéma, Paris, Éditions du Chêne, 1945, p. 10.

20 Voir Lynn Kirby, Parallel Tracks : The Railroad and Silent Cinema, Durham, Duke U.P., 1997.

21 Ricciotto Canudo, op. cit., p. 189.

22 René Doumic, "l'Âge du cinéma ", la Revue des Deux Mondes, 15 août 1913, p. 927. 
interne du corps des spectateurs : car les mouvements frénétiques perçus à l'écran produisent de l'activité nerveuse et musculaire chez le spectateur.

L'impact de la forme, du mouvement et de la couleur sur le corps (et pas seulement sur l'œil) a été mesuré à partir des années 1880 en France. Le psycho-physiologiste Charles Henry a démontré (des centaines d'expériences à l'appui) que la direction des mouvements est tantôt dynamogène (stimulante), tantôt inhibitrice et qu'à l'instant même où nous percevons un objet, notre « machinerie interne " le reproduit. En 1885, il écrit que, grâce aux mouvements oculaires et aux transformations produites dans nos systèmes vasculaire, musculaire et respiratoire, " il n'y a pas d'idée sans mouvement virtuel, puis réel ${ }^{23}$. Théodule Ribot a commencé son Essai sur l'imagination créatrice de 1900 en déclarant que l'une des conquêtes principales de la psychologie contemporaine « est d'avoir solidement établi l'importance des mouvements, et d'avoir démontré que la représentation d'un mouvement est un mouvement qui commence ${ }^{24}$. Appliquant l'esthétique scientifique au cinéma, Édouard Toulouse et Raoul Mourgue ont écrit au début du XXe siècle :

Étant scientifiquement démontré que la perception du mouvement fait naître l'ébauche du mouvement correspondant, il se produirait à l'écran un phénomène du même genre que la suggestion hypnotique pratiquée après avoir mis le sujet dans une attitude donnée. ${ }^{25}$

Si le spectateur de caf'conc' ou du cinéma burlesque éprouve dans son corps de l'excitation dynamogénisante, il éprouve aussi de l'anxiété à sentir son corps imiter involontairement les mouvements et les grimaces hystériques si fréquemment décrits dans la presse. Ce style de performance provoque angoisse et hilarité, la seconde en réaction à la première. Henri Bergson écrit : « la débilité mentale et toutes les variétés de folie nous rendent mal à l'aise en présence de leurs symptômes mécaniques. Le rire est un effort pour corriger ces défauts ${ }^{26}$. Ou encore : « Lorsque la société est en présence de quelque chose qui l'inquiète [...] c'est donc par [le rire] qu'elle y répondra ${ }^{27}$. C'est la raison pour laquelle les difformités, les dislocations, les tics et les convulsions épileptiques sont si drôles aux yeux de bien des spectateurs. En 1921, Jean Epstein explique pourquoi le cinéma tire un si grand profit des symptômes mécaniques. Et ce n'est pas dans le seul but de faire rire le spectateur :

23 Charles Henry, Introduction à une esthétique scientifique, Paris, Hermann, 1885, p. 2.

24 Théodule Ribot, Essai sur l'imagination créatrice, Paris, Alcan, 1900, p. 1.

25 Dr. Edouard Toulouse et Raoul Mourgue, « Des réactions respiratoires au cours de projections cinématographiques ", rapport présenté à l'Association française pour l'avancement des Sciences, 1920. Cité dans Léon Moussinac, Naissance du cinéma, Paris, Éditions d'Aujourd'hui, 1983 [1925], p. 174. 26 Henri Bergson, op. cit., p. 100 (je souligne).

27 Ibid., p. 15. 
Le metteur en scène suggère, puis persuade, puis hypnotise. [...] la pellicule n'est qu'un relais entre la source d'énergie nerveuse et la salle qui respire son rayonnement. C'est pourquoi les gestes qui portent le plus à l'écran sont des gestes nerveux. Le nervosisme, qui exagère souvent les réactions, [est] photogénique. Chaplin a créé le héros surmené. Tout son jeu est en réflexes de nerveux fatigué. Une sonnette ou un clakson [sic] le font sursauter, le dressent debout et inquiet. [C'est] un synopse de neurasthénie photogénique.28

Si l'essence du cinéma est la vélocité et le mouvement perpétuel comme le croyaient Doumic et Canudo, alors la rapidité et la nervosité des gestes (qui incluent les gestes automatiques des maladies nerveuses) seraient une forme spécifique de l'essence cinématographique. Ici un interne chez le spectateur. Que ce soient les chocs reçus des gestes saccadés ou ceux, encore plus forts, de l'agitation convulsive hystérique, il s'agit d'une expérience corporelle des plus intenses. Pour résumer, le ressenti si intense de la réalité de l'image filmique est dû en grande partie aux réactions automatiques des nerfs et des muscles du spectateur, aussi bien qu'aux mouvements oculaires. Cette compréhension du spectatorship qui me vient de l'étude de la psycho-physiologie et de la neurologie du XIXe siècle se justifie par la présence de ces mêmes notions dans le discours journalistique autour du cinéma entre 1908 et $1930^{29}$, y compris dans la presse populaire. Les études des vingt dernières années sur les neurones-miroir ${ }^{30}$ viennent d'ailleurs confirmer le bien-fondé des travaux d'Henry et de ses collègues, évoqués ici.

La vélocité entretient une relation privilégiée avec le film comique : en effet, elle en est l'un de ses principaux moteurs. Il y a la réaction au quart de tour, I'accélération puis l'effet en domino des séquences de chaos et démolition dans les comédies anarchiques, le truc du mouvement accéléré tout court, la gestuelle et le mouvement épileptique... sans parler de la poursuite comique. Canudo a bien vu que le Cinématographe rendait possible « un type

28 Jean Epstein, « Grossissement », Bonjour Cinéma, Paris, Éditions de la Sirène, 1921, pp. 101-102. Le nervosisme est un état avant-coureur de I'hystérie (voir l'article de vulgarisation du Dr. Jules Rengade, " Le Nervosisme ", le Courrier Français, 5 juillet 1903, pp. 10-11).

29 Epstein, qui avait étudié la médecine, expliquait le fait que le ressenti cinématique est particulièrement intense par une "modifi[cation] du fonctionnement du système nerveux » (ibid., p. 107). Le psychologue Hugo Münsterberg ne manquait pas non plus de voir les effets du film sur le spectateur à travers ce prisme. Voir son The Photoplay, a Psychological Study, New York, Appelton \& Co., 1916. La dette de Walter Benjamin envers la psycho-physiologie me semble assez claire. Voir l'article magistral de Miriam Bratu Hansen, "Benjamin and Cinema ", Critical Inquiry, mars 1999, pour une analyse du concept d'innervation mimétique utilisé par Benjamin dans le contexte du cinéma.

30 Ce sont les neurones dans le cortex visuomoteur du singe qui déchargent à la vue d'une action faite devant lui. Des expériences chez l'humain (y compris devant la projection de vidéos) suggèrent fortement que les propriétés similaires dans le circuit fronto-pariétal sont présentes. Ces expériences sont faites depuis 1996 et sont reprises dans Giacomo Rizzolatti et Corrado Sinigaglia, les Neurones miroirs, Paris, Odile Jacob, 2008. 
comique nouveau (...) [en ajoutant] l'élément de la rapidité absolument précise ${ }^{31}$. Une variation bien intéressante de la poursuite en ligne droite est celle en zigzag que l'on peut voir dans les films de Méliès et de Segundo de Chomon. Cette chorégraphie de corps qui dessinent des zigzags en changeant de direction (de gauche à droite et l'inverse, ainsi que de bas en haut et l'inverse) produit une réaction physiologique encore plus forte sur le spectateur dont la « machinerie interne " reproduit ces changements abrupts de direction 32 .

Il y aurait un genre de changement de sens similaire à l'œuvre dans certains processus pour produire le rire. Les études psycho-physiologiques du rire, à commencer par celles de Herbert Spencer et James Sully en Angleterre, puis par Ribot et Ludovic Dugas en France, décrivent le trajet que prennent les réactions nerveuses et musculaires pour aboutir au rire. Prenons le cas de l'effet comique qui vient d'une attente trompée. La dynamique est la suivante : une grande quantité d'énergie nerveuse est subitement arrêtée dans son cours. L'excès doit se décharger dans une autre direction via les nerfs moteurs et les muscles : cette décharge semi-convulsive est le rire. Une autre dynamique consiste en une tension musculaire composée de chocs qui aboutit à une décharge. Cette dynamique est considérablement augmentée par la vélocité des chocs.

Une autre forme de vélocité se trouve dans les films d'animation d'Émile Cohl. Dans le Retapeur de cervelles de 1910 (film composé en partie de dessins et en partie de prises de vue " réelles »), on guérit un patient en lui enlevant ce que le scénario nomme " l'enchaînement de ses idées folles ». Un vieux couple entre dans le cabinet du Dr. Trépanoff ; I'homme est travaillé par des tics et un tremblement - les symptômes de l'hystérie masculine et de la neurasthénie. Le pauvre homme mord la poignée de sa canne pendant que le docteur place un objet qui ressemble à un mégaphone sur sa tête. Le film passe ensuite au dessin animé, et c'est alors que la fête commence. Cohl va littéralement nous montrer ce qui se passe à l'intérieur du cerveau. On y voit d'abord un insecte, puis cinq têtes différentes, puis un cosaque dansant, parmi plusieurs autres transformations. Or, ce tableau changeant de la psyché du patient n'est que le début de métamorphoses absolument vertigineuses. Cohl retourne aux acteurs en chair et en os pour montrer le médecin qui va chercher une vrille avec laquelle il fait un trou au crâne... ce qui lui permet d'extraire un long ver blanc qu'il met sur un tableau noir. Retour à l'animation : on regarde la ligne blanche de cette lésion cérébrale opérer des transformations d'une rapidité étonnante, figurant des fantasmes d'incorporation, de dédoublement, de démem-

31 Ricciotto Canudo, op. cit., p. 196. Emmanuel Dreux (op. cit., p. 33) cite cette remarque de Canudo, ainsi que l'élément primordial des déformations dans la définition du « très comique » chez Canudo.

$32 \mathrm{Et}$ si on commercialisait un jeu vidéo WI (qui incorpore nos gestes à l'écran) proposant non le tennis ou le golf, mais des films burlesques? 
$1895 /$

\section{Les automatismes du Double}

Rappelons que l'idée dominante du Rire d'Henri Bergson est l'automatisme envisagé comme source du comique. "Le comique est inconscient [...] le geste échappe ; il est automatique "35. "Les attitudes, gestes et mouvements du corps humain sont risibles dans l'exacte mesure où ce corps nous fait penser à un simple mécanisme »36. Et en miroir : « nous adoptons les gestes et les difformités risibles de l'automate qui nous fait rire " ${ }^{37}$. Non seulement il insiste sur le rôle des automatismes à la fin de la décennie où la psychiatrie en parle tant, mais au moment même où la popularité du café-concert et de son successeur le music-hall sont à leur apogée. Quelle variété de folie Bergson trouve-t-il la plus pertinente à l'esprit du comique ? Celle que l'on trouve très souvent dans les rêves : I'aliénation d'une partie de soi-même dans un Autre, lorsqu'on se regarde dédoublé de l'extérieur ${ }^{38}$. Cette partie aliénée peut en effet prendre des formes très oniriques, celles que Freud va étudier en 1919 sous les auspices de I'Inquiétante étrangeté (Das Unheimliche).

33 Dr. Paul-Max Simon, Sur I'hallucination visuelle, Paris, Baillière, 1880, p. 15.

34 Donald Crafton, Emile Cohl, Caricature, and Film, Princeton, Princeton University Press, 1990, pp. 200 et 272 .

35 Henri Bergson, op. cit., p. 109.

36 Ibid., pp. 22-23, en italiques dans le texte.

37 Ibid., p. 109 (je souligne).

38 Laurent Le Forestier retrouve les points essentiels de l'essai de Bergson dans le cinéma burlesque ; il souligne également la dette de cette esthétique aux formes de spectacle qui l'ont précédé. Voir « le Cinéma français des premiers temps, comique par essence ? ", le Genre comique, Christian Rolot et Francis Ramirez (dir.), Montpellier, Centre d'Étude du XXe siècle / Université Paul-Valéry (Montpellier III), 1997. 
Freud y étudie surtout le caractère unheimlich inhérent au Double, mais commence par les exemples du phénomène proposés dans un article de 1906 d'Ernst Jentsch. Les figures de cire, les poupées, les automates, la catalepsie, et l'épilepsie - auxquels Freud rajoute les membres ou têtes détachés, et les pieds qui dansent tout seuls - sont tous des formes de la même perception unheimlich : un objet inanimé qui devient vivant, instaurant le doute s'il s'agit d'un être vivant ou d'un automate. De plus, «l'effet unheimlich des crises épileptiques et de la folie [vient de] l'impression des processus automatiques qui pourraient bien se dissimuler sous l'apparence ordinaire de l'activité mentale ${ }^{39}$. De ces forces automatiques exhibées dans l'épilepsie, et qui ne semblent pas appartenir au monde humain selon Jentsch, Freud nous dit en effet que le spectateur de la crise « y voit la manifestation de forces qu'il ne soupçonnait pas chez son prochain, mais dont il peut pressentir obscurément l'existence dans des recoins les plus reculés de sa propre personnalité ${ }^{40}$. On voit à quel point ces idées collent parfaitement avec les figures souvent représentées au cinéma burlesque, ainsi qu'avec la théorie de l'inconscient corporel. En effet, la notion d'Unheimlich - étranger mais cependant familier - signifie la sensation que nous avons du Double à l'intérieur de nous, notre inconscient corporel avec ses gestes automatiques qui expriment un programme dont on préfère ne rien savoir. Enfin, une dernière catégorie d'inquiétante étrangeté devrait également faire penser à l'expérience du spectateur du cinéma des premiers temps, par exemple dans les vues fantastiques de Méliès : c'est lorsque quelque chose que l'on a considéré comme imaginaire se manifeste dans la réalité.

Contemporain du Rire de Bergson et de l'article de Jentsch, Pierre Janet écrit qu'il trouve " un sentiment de dédoublement plus ou moins accentué chez tous mes malades » et qu'il pouvait en présenter une centaine d'exemples ${ }^{41}$. Combien de films comiques dans la période qui nous intéresse ici montrent ce Double anarchique et primitif ?42 Les dédoublements, comme les métamorphoses, peuvent être obtenus avec une force inédite au cinéma par la surimpression, par les caches, par les fondus, par le trucage par arrêt de manivelle et substitution, ou par collure... trucages que Méliès a justement désignés comme « tout l'arsenal des compositions fantaisistes et abracadabrantes à rendre fou [sic] les plus intrépides ${ }^{43}$.

39 Sigmund Freud, I'Inquiétante étrangeté, Paris, Interférences, 2009, p. 25 ; traduction de Marie Bonaparte et E. Marty, modifiée par I'auteur d'après la traduction en anglais.

40 lbid., p. 80.

41 Pierre Janet, les Obsessions et la psychasthénie, Paris, Alcan, 1903, p. 312.

42 Quant au Double comme miroir du spectateur, Jean Epstein écrit que « dans son double à l'écran, ce que le spectateur remarque d'abord, [c'est] cette vulgarité d'une attitude [...] qu'il avait cru réussir à cacher. " (I'Intelligence d'une machine, Paris, Melot, 1946, p. 107. En 1907), Giovanni Papini avait écrit que ces images de " nous-mêmes » nous font découvrir « le ridicule de certains gestes mécaniques ». ("La Philosophie du cinématographe », dans Daniel Banda et José Moure, op. cit., p. 138).

43 Georges Méliès, "Les Vues Cinématographiques », Annuaire général et international de la Photographie, Paris, Plon, 1907, p. 370. 
$1895 /$

Otto Rank a bien vu en 1914 que le cinéma pouvait montrer les pathologies mentales que la littérature peinait à décrire ${ }^{44}$. Quant à André Deed, il se passe des trucages dans Boireau et la gigolette, qui s'ouvre avec notre héros jouant aux cartes comme s'il y avait quelqu'un en face, n'hésitant ni à regarder les cartes de cet autre, ni à l'engueuler. Ce dédoublement déconcertant campe bien le personnage qui, peu après, tourne mécaniquement comme une toupie à deux reprises en sortant de chez lui.

On retrouve la notion du dédoublement dans d'autres théories du rire autour de 1900, un phénomène surtout en évidence lorsqu'on rit de soi-même. On pourrait arguer aussi que le rire du spectateur devant les pathologies corporelles fait de lui le miroir de ces mêmes pathologies. La ressemblance du rire aux convulsions est notée par maints psychologues, physiologistes, philosophes et anthropologues de l'époque, et Herbert Spencer a même démontré que le rire intellectuel suit le même processus physiologique que le fou rire convulsif. Le psychologue Ludovic Dugas cite un sonnet : « Mais quel est ce cas d'épilepsie, [...] les nerfs détraqués ? C'est le Rire ${ }^{45}$. De plus, le rire est contagieux, "propagé par l'action réflexe »46. Certains rires sont involontaires et quelqu'un pris par le fou rire " devient une espèce de curiosité ${ }^{47}$. En d'autres mots, le fou rire nous réduit au même état que le personnage sujet aux gestes convulsifs et automatiques qu'on est en train de regarder à l'écran. Enfin, le rire violent est un surgissement « de la vie animale, [tout comme] le rire gras est de l'animalité qui était refoulée ${ }^{48}$.

Les études sur le rire entre 1885 et 1900 sont presque toutes d'accord pour dire que la perception d'une difformité déclenche toujours le sentiment du comique ${ }^{49}$. Le rire est un effort pour corriger le défaut, ou bien on rit devant la difformité ou la débilité d'autrui parce qu'on se sent supérieur. Selon Dugas, plus on manque de raffinement, plus le rire est brutal. Ce rire appartient aussi bien aux enfants et aux sauvages (notons que, d'une manière ou d'une autre, il signale un manque d'évolution) : « Ces gens auront le cœur de rire du tortillement d'un bancal, de la laideur d'un bossu, de la hideur repoussant d'un idiot " 50 . II s'avère que ces trois objets de dérision correspondent à des attractions très populaires au caf'conc' et dans le cinéma comique entre 1898 et 1912. Le rire est brutal, à mon sens, parce que le sentiment de supériorité n'est ressenti qu'au niveau conscient ; la peur superstitieuse et identificatoire fait vaciller sa solidité. Une Angst de se voir dans ces figures comme dans un miroir déformant produit

44 « Der Doppelgänger » (le Double), Imago, n³, 1914.

45 Ludovic Dugas, la Psychologie du rire, Paris, Alcan, 1902, p. 122.

46 Ibid., p. 36.

47 Ibid., pp. 24-25.

48 Ibid., p. 125.

49 Alfred Michiels, le Monde du comique et du rire, Paris, Calmann Lévy, 1886, p. 159.

50 Ludovic Dugas, op. cit., p. 98. 
un sentiment de malaise surgi de l'inconscient et qu'il faut à tout prix évacuer par le rire ${ }^{51}$. Les difformités physiques et mentales fascinaient le public, d'où la quantité de Phénomènes exhibés dans les foires, et parfois au café-concert. Comme une coupure de presse intitulée le Temps des Monstres nous informe,

Un monstre fut jadis un objet d'horreur, de répulsion. Maintenant c'est l'un des moyens de devenir riche et célèbre [...] grâce surtout à la curiosité hystérique de la foule, très affamée de ces exhibitions sensationnelles. [On est] transporté dans une vie hideuse, peuplée d'étonnements comiques et $d^{\prime}$ êtres cauchemardesques. 52

La répulsion devant des êtres oniriques, hideux, attire les spectateurs fascinés, et elle se convertit en une réaction d'étonnement comique. Dynamique qui n'est pas si éloignée de celle à l'œuvre dans les films burlesques qui plongent le plus bas dans le royaume du Low. Les figures absolument fantasques dans la Dent de Margottin d'Alfred Machin (Nizza, 1912) nous en donneront une idée.

La rage de dent de Margottin est tellement aiguë dans la première séquence qu'il se propulse contre le mur de sa chambre, puis attache sa dent dans le but de l'extraire à une corde pendue du plafond. Deux amies rentrent et voient Margottin par terre, contorsionné comme par une crise épileptique. Ces femmes sont grimées à outrance, l'une, extraordinairement grande, est en blackface avec de surcroît un masque carnavalesque. Elles essayent de traîner son corps, soudain rigide comme s'il était en catalepsie, pour l'amener chez un dentiste, mais il se met à hurler, à donner des coups de pied, et à se démener dans tous les sens ; l'agitation et l'abandon extrêmes avec lesquelles se déplacent et gesticulent ces personnages sont vraiment impossibles à décrire. La grande femme (un homme travesti) - plus une créature sauvage qu'une femme - agit avec autant de folle énergie que Margottin. Chez le dentiste, il saute brusquement du fauteuil et se catapulte à travers le mur, détruisant le cabinet ; ensuite il court de plus en plus frénétiquement à la recherche d'un remède (je passe sur ces scènes surréalistes), mais sans succès. Enfin, les amies lui mettent un pétard dans sa bouche qui fait exploser toutes ses dents, et on le voit grimaçant avec plaisir dans le gros plan de la fin.

51 Alfred Jarry a expliqué la réception d'Ubu roi ainsi : face à cette figure de monstruosité burlesque et terrifiante à la fois, " le public [a été] stupéfait à la vue de son double ignoble. » (Jarry parle ici de l'échec de la pièce, mais n'oublions pas que l'attente du public au Théâtre de l'Geuvre différait considérablement de celui du cinéma burlesque.) Alfred Jarry, "Questions de théâtre ", la Revue blanche, 1er janvier 1897, repris dans A. Jarry, Tout Ubu, Paris, Librairie Générale Française, Livre de poche, 1962, p. 153.

52 Coupure de presse sans date dans le dossier « Phénomènes ", BnF Arts du spectacle RO 17445, p. 3. Citons Jean-Louis Schefer : "L'aberration anatomique est telle qu'elle fait songer au burlesque, en cela que le corps s'excède dans sa difformité " (cité dans Jean-Philippe Tessé, le Burlesque, Paris, Petits Cahiers du cinéma, 2007, p. 88). 
Revenons maintenant aux études psychiatriques avant 1900, car il nous faudra préciser le rôle important du somnambulisme et dans la découverte de l'inconscient, et dans les films comiques.

\section{Imitation et somnambulisme}

Si la clé de l'inconscient a été fournie par l'observation de patients en état somnambulique, c'est parce qu'on y témoignait la manifestation d'une autre personnalité, faite pour la plupart de sensations et d'automatismes. Les expériences faites sur les hystériques sous hypnose font surgir une deuxième personnalité " cachée " sous la première. Ce schisme entre la conscience et l'état second, comprise comme un dédoublement de la personnalité, se révèle être une division entre la conscience et l'inconscient. II est important de souligner que la " deuxième personnalité » se permettait des actes et des gestes en très grand contraste avec le comportement bien plus sage de la première.

Les images d'hystériques en poses cataleptiques sous hypnose étaient fort répandues à partir de 1878 , et la perte totale de volonté qui allait de pair avec cet état était parfaitement connue (sinon grâce à la Salpêtrière, alors par le biais de magnétiseurs ambulants ou par la presse populaire). Cet arrière-fond accompagne les spectateurs au cinéma. Les somnambules sont nombreux dans les films français, et cela à partir de 1897 avec le Magnétiseur de Méliès. Cependant, le premier film à ma connaissance à poser explicitement la métaphore hypnotisme et imitation inconsciente = cinéma est le Miroir magique ou le Miroir hypnotique de Louis Feuillade (Gaumont, 1909). On y dévoile une invention qui investit l'image filmique de pouvoirs magnétiques : une boîte contenant un miroir-écran capable de lire dans le cerveau, de mettre la personne visée en transe somnanbulesque et lui faire répéter devant nous les actes transgressifs qu'il a cachés ${ }^{53}$. Actes qui sont simultanément vus sur le petit écran. Les trois films dans le film sont tous des projections de la double personnalité : criminelle, anarchique ou libidineuse. Les moments comiques dans ce film résultent du choc de surprise du spectateur qui voit réapparaître dans la petite boîte une scène déjà perçue précédemment. La surprise est mirée par les autres personnages dans le film, spectateurs comme nous. Le deuxième motif du rire vient du spectacle d'un corps inconscient répétant des gestes automatiquement devant l'écran (fig. 4). Nombreux étaient ceux qui croyaient que l'image filmique suggestionnait le spectateur, et que I'expérience du spectateur était une forme d'hypnose ${ }^{54}$. Jean Epstein parle même d'une « faim

53 Le Binettoscope d'Émile Cohl (Gaumont, 1909), met en scène un autre moyen d'incorporation. Un clown (pour le moins troublant) explique aux spectateurs qu'il va les faire apparaître sur l'écran d'une boîte-caméra de son invention, le binettoscope. Tel un magnétiseur, il attire par des passes le visage. 54 Voir la citation très connue de Frank Woods sur « l'illusion hypnotique qui [s'empare] du spectateur, le retenant par le pouvoir de la suggestion » (New York Dramatic Mirror, 14 mai 1910). 


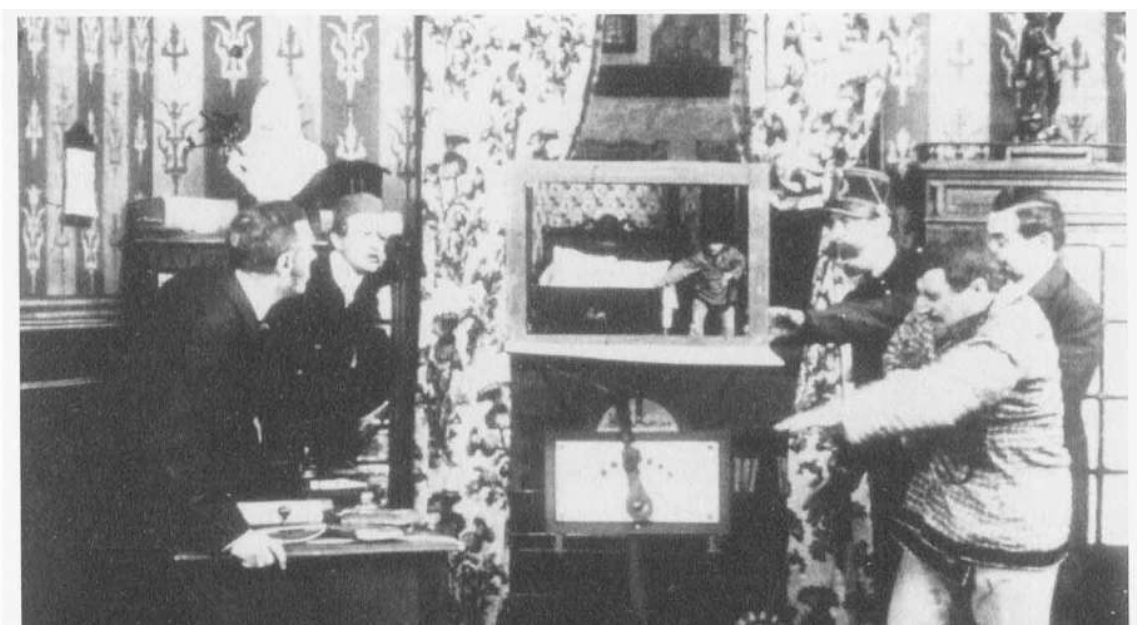

Fig. 4. Le Miroir hypnotique, Gaumont, 1909.
$1895 /$ $n^{\circ} 61$ septembre 2010 hypnotique pratiquée après avoir mis le sujet dans une attitude donnée " (je souligne). Cependant, c'est surtout le corollaire de la suggestion, l'imitation, qui nous intéresse ici. En effet, ce miroir hypnotique qui ressemble à s'en méprendre au cinématographe évoque la répétition inconsciente interne des spectateurs. Les mises en abyme des films dans le film seraient donc à double tranchant.

Au moment où les psycho-physiologistes démontrent que la perception d'un mouvement entraîne un mouvement interne correspondant, les psychiatres conviennent que l'instinct $d$ 'imitation " semble venir des parties inconscientes du système nerveux " ${ }^{56}$. Tout le monde est suggestible selon les psychologues de l'École de Nancy (Bernheim, Liébeault). Pour le sociologue Gabriel Tarde, qui s'inspire de ces travaux en psychologie, la suggestion et son corollaire l'imitation inconsciente sont un phénomène universel : « Rien de plus vulgarisé que cette vue ${ }^{57}$; ou encore : «Une action nous donne [...] l'idée plus ou moins irréfléchie

55 Jean Epstein, « Grossissement », op. cit., p. 107.

56 Selon le Dr Paul-Max Simon, « il ne fait aucun doute que l'épilepsie peut être engendrée par imitation, aussi il faudra veiller à ce que des enfants, des jeunes gens, des femmes impressionnables ne soient jamais témoins de crises ". (Hygiène de l'esprit au point de vue pratique des maladies mentales et nerveuses, Paris, Baillière, 1881, pp. 25 et 37).

57 Les Lois de l'imitation, Paris, reprint Kimé, 1993 de l'édition de 1890, p. 84. Tarde conclut que si l'imitation inconsciente en réponse à une suggestion est un phénomène universel, alors la figure paradigmatique de l'être humain vivant en société est le somnambule. 


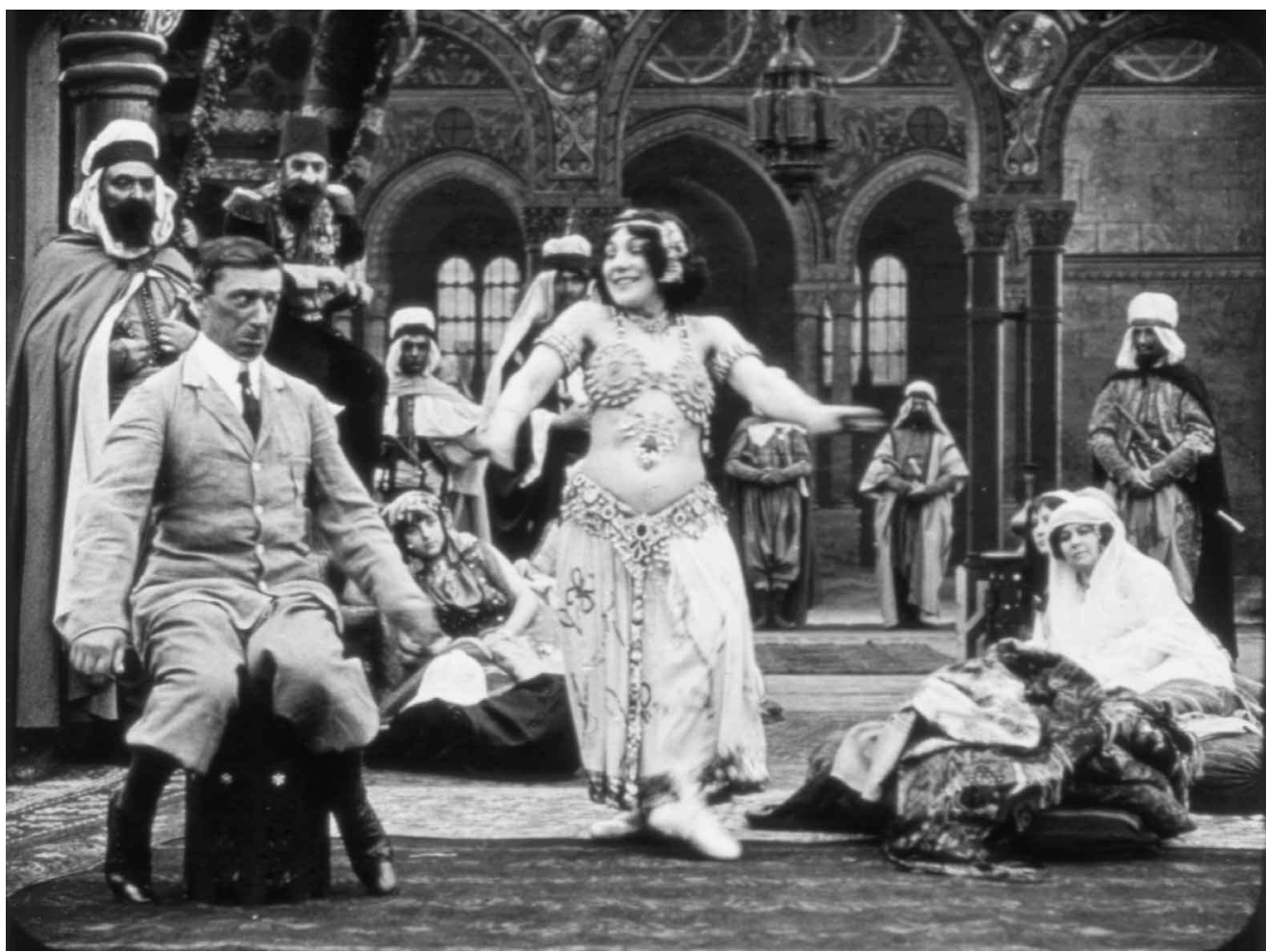

Fig. 5. Boireau au harem, Pathé, 1912.

de l'imiter "58. Cette théorie est illustrée par maints films burlesques. Par exemple, Deed est photographe à Istanbul dans Boireau au harem (Pathé, 1912). Spectateur d'une danse du ventre au harem, il imite irrésistiblement les mouvements de la danseuse (fig. 5). II va ensuite être obligé de se déguiser en danseuse, et de faire la danse du ventre dans I'une des scènes les plus hilarantes du cinéma burlesque. Dans Little Moritz demande Rosalie en mariage (Comica, 1911), Moritz, assis à côté du père de Rosalie, saute en l'air chaque fois que ce dernier frappe sur son bureau en guise de réponse négative à la demande de mariage. Spectatrice derrière la porte, Rosalie saute à chaque fois aussi à la vue de Moritz.

Vingt ans après les Lois de l'imitation, Louis Haugmard accuse le film de " produi[re] des spectateurs muets et hypnotisés [qui perdent] toute volonté de raisonner et de construire. [...] Et ce sera un notable symptôme de fin de race ». Le film « déclenche l'imitation car l'image excite les âmes naïves. Ainsi, « le Cinématographe [...] ce sera de l'action pour neurasthéniques »59.

58 Ibid., p. 87.

59 Le Correspondant, 25 mai 1913, pp. 768-770. Notons que fin de race signifie dégénérescence. 
Même les défenseurs du cinéma comme Hugo Münsterberg admettaient que la force des impressions sur l'esprit étaient « le point du départ de l'imitation et d'autres réponses motrices. "60 Or, ce sont justement des "sensation[s] affective[s] d'ordre nerveux "61 que le cinéma préconisé par Antonin Artaud doit capter pour « agir sur la matière grise du cerveau directement $» 62$. Et quel genre cinématographique y réussit le mieux ? Le film comique :

[II] retrouve comme la disposition primitive des choses [...] comme les premiers Malec63, comme les Charlot les moins humains. Le cinéma constellé de rêves, et qui nous donne la sensation physique de la vie pure, trouve son triomphe dans l'humour le plus excessif [et] se traduit bien dans les convulsions et les sursauts d'une réalité qui semble se détruire elle-même avec une ironie où I'on entend crier les extrémités de l'esprit. 64

Voici un film onirique, convulsif, et cruel, en somme, très artaudien. Dans Onésime contre Onésime de Jean Durand (Gaumont, 1912), le Double fonctionne comme paradigme de l'imitation, des automatismes et de l'expérience d'incorporation chez le spectateur. Le dédoublement est ici clairement assimilé à la division entre les ordres supérieur et inférieur. Le premier intertitre nous apprend que « le bon Onésime savait se conduire dans le monde, mais le mauvais s'y tenait comme un voyou ». La deuxième personnalité est pur libido, appétit insatiable, et anarchiste. Par exemple, il caresse les fesses de la cuisinière et vole les couverts dans un restaurant. Le second Onésime surgit et se détache à chaque fois du corps du premier par surimpression. Cette technique, souvent utilisée pour représenter le moi onirique ou somnambule, rend à merveille ici l'image du dédoublement et du surgissement du moi instinctuel venant des facultés inférieures. Genoux pliés, fesses en l'air, avec son visage narquois de clown, le double fait une grimace à la caméra. "Dégoûté » de son alter ego, le bon Onésime " se fâcha et se sépara de l'autre "; il y parvient en quittant son lit par une pirouette pour laisser derrière lui son double instinctuel. Mais « le mauvais Onésime en profita pour se conduire encore plus mal encore ". Il imite les gestes de son " bon moi », de sorte qu'on ne peut plus le distinguer de son double respectable. Le bon Onésime furieux grimace à son tour vers la caméra comme I'avait fait son double, et veut s'échapper mais l'autre le poursuit, rampant à un moment par terre comme un animal. La division et l'incorporation figurées jusqu'ici grâce à la surimpression sont le clou de la scène finale, et là l'incorporation est représentée de façon littérale. De retour à la maison, et hors de lui (c'est le cas de le dire), le « bon » Onésime jadis civilisé

60 H. Münsterberg, op. cit., p. 221.

61 Antonin Artaud, "Cinéma et réalité », Guvres complètes, T. III, Gallimard, 1978, p. 18.

62 Antonin Artaud, "Réponse à une enquête ", ibid., p. 74.

63 Le nom du personnage de Keaton entre 1920 et 1923.

64 Artaud, "Cinéma et réalité », ibid., p. 20. 
$1895 /$

démembre le méchant Onésime et, comme nous le dit l'intertitre, " le mangea " ! Voici comment : son regard devient méchant et ses mains se contractent automatiquement ; il s'empare de son double assoupi dans un fauteuil et, enragé, lui déchire un bras, puis l'autre, les jambes, le torse avec la tête, les jetant dans I'air. Soudain, une idée lui vient à l'esprit : il nous sourit et salue avec son chapeau. Le moment $d$ 'incorporation par dévoration manque dans la copie du CNC, mais le regard à la caméra dans le dernier plan signifie assez que le spectateur est bien mis en connivence avec ce moment ultime ${ }^{65}$. Ce film, tel les autres films burlesques discutés ici, met en scène le retour du Primitif, avec la régression d'un corps automatique libéré des facultés supérieures, ce corps déchaîné qu'on peut voir aussi bien à la Salpêtrière que dans le spectacle forain et dans le cinéma burlesque. Cinéma primitif s'il en est.

Comme dans le Miroir hypnotique, la Photographie électrique à distance de Méliès (1908) explore le pouvoir d'une invention qui permet de voir la deuxième personnalité d'un individu. Les photographies, qui sont d'abord immobiles, se mettent en mouvement lorsque la personnalité cachée émerge. Posant devant la caméra, le mari d'un vieux couple devient un singe et sa femme une maniaque grimaçante. En janvier de la même année, Boireau personnifie un singe dans deux films qui donnent libre jeu à l'imitation : "Lors d'une [...] trépanation, le chirurgien se trompe et remplace la cervelle du patient par celle d'un singe [qui] imite les gestes des autres " (I'Homme-singe, Pathé, 1909). Notons que l'affiche montre un homme avec la tête d'un singe. Dans l'Esprit d'imitation (Pathé, 1909), "il s'ingénie à des imitations aussi grotesques que variées " ${ }^{66}$. L'un des grands sujets de la presse en janvier 1909 était le nouveau chaînon manquant néandertal qui venait d'être découvert en France six mois auparavant. La présence des singes dans les films de Méliès est également à noter, par exemple dans le Savant et le chimpanzé (1900). Avec la dissémination du darwinisme parmi le grand public, le singe souligne la portée régressive du genre de comportement et de mouvement illustrés dans le burlesque.

\section{Conclusion}

Un dernier film burlesque, situé au café-concert et placé sous l'égide de la pulsion d'agressivité, offre des images bien chargées d'inquiétante étrangeté. Tom Tight et Dum-Dum de Méliès (1903) contient bon nombre des éléments recensés jusqu'ici, à commencer par la domination totale des facultés inférieures sur les facultés supérieures. Il fait boucle avec les genres

65 Un autre exemple de cannibalisme : dans Boireau, bonhomme de pain d'épice, Boireau tombe dans le four du boulanger, et la dernière image est un gros plan de deux petites filles en train de manger l'énorme bonhomme de pain d'épice qu'il est devenu (Pathé, 1913; fig. 6). L'incorporation représentée ici sert-elle de miroir à notre incorporation de l'image filmique ?

66 Henri Bousquet, Catalogue Pathé des années 1896-1914, vol. 2 (1907-1909), Paris, H. Bousquet, 1993, p. 147. 


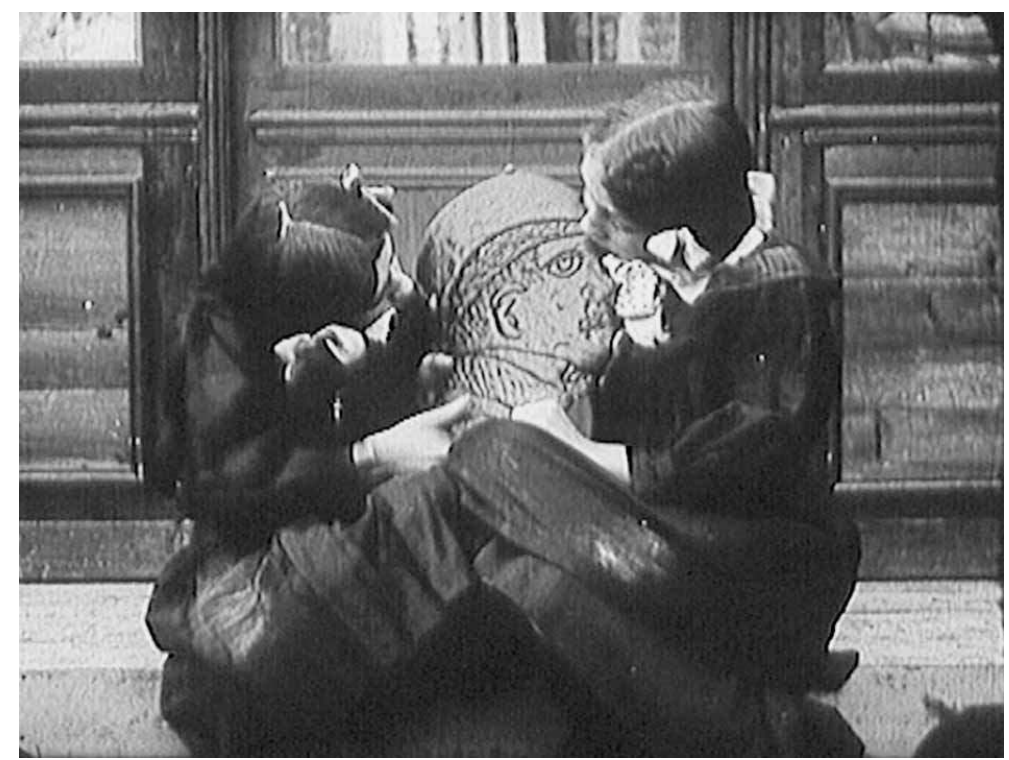

Fig. 6. Boireau bonhomme de pain d'épice, Pathé, 1913.
$1895 /$ $n^{\circ} 61$ septembre 2010

de performance au café-concert discutés au début de cet essai, car le personnage central est un comique excentrique, quasi-synonyme de comique épileptique ${ }^{67}$. II est inquiétant à un degré rarement trouvé dans les films de Méliès. Sans doute parce que l'illustration très violente du déchaînement des pulsions est plus crue ici, et pas atténuée par la grâce infinie des évolutions véloces de Méliès même lorsqu'il se déplace frénétiquement.

Tom Tight et Dum-Dum offre une exhibition de pathologies nerveuses, à commencer par l'agitation frénétique, puis par le fantasme de démembrement, et l'impossibilité de rester assis (récemment observé par Pierre Janet chez certains de ses patients). Le film commence avec l'entrée en scène du comique, un personnage à ventre énorme, portant trois parties d'une poupée de taille humaine : la tête, le torse avec bras, et les membres inférieurs. Il les rassemble et les place sur une chaise. Lorsqu'il quitte la scène momentanément, un chanteur entre et commence son numéro. Le comique revient en exécutant des pas de danse ultrarapides quand, soudain, il aperçoit l'autre artiste. Il tape sur le chanteur mais ce dernier continue, imperturbable, à chanter. Le comique trépigne, saute, gesticule comme un fou, court à gauche pour prendre un tabouret, s'y assoit pendant deux secondes et regarde, puis prend un bâton et court l'asséner sur la tête du chanteur (sans effet), l'asperge d'eau (sans effet),

67 Le genre était encore très prisé au café-concert et au music-hall jusqu'en 1908. 
$1895 /$

puis à l'aide d'un maillet géant, enfonce l'autre sous les planches jusqu'à la taille et toujours chantant. Il s'agit de l'effet comique de la persévérance dont parle Jean-Philippe Tessé : la mécanique du personnage burlesque - une fois mise en marche - ne peut plus être arrêtée, car le chanteur poursuit sa performance malgré les coups. Le comique prolonge son action jusqu'à ce que la tête seule dépasse le sol... et cette tête continue de chanter ! Furieux, le héros l'enfonce complètement avec des coups de pieds, va s'assoir dans une pose exagérée, les jambes raides et avec un mouvement automatique de la jambe gauche. Deux assistantes lui lancent la tête de la poupée, qu'il embrasse, puis jette violemment par terre. Elle rebondit comme un ballon, il l'attrape, la relance, la saisit au bout d'un bâton, puis sur sa propre tête... toujours agité, courant - ou plutôt se jetant - dans tous les sens en donnant des coups de pieds automatiques d'une façon qui défie la description verbale. II place toutes les parties de la poupée sur le tabouret et les recouvre d'un drap ; elle se transforme en ballerine, car le comique épileptique est également un illusionniste. Imitant les pirouettes et sauts de la danseuse, le ventripotent l'attrape et elle retombe en morceaux. Entretemps, le chanteur émerge de la trappe ; son rival le jette en l'air en petits morceaux - qui retombent sur le corps morcelé de la danseuse, à la suite de quoi le comique leur saute furieusement dessus, les écrasant, puis pour parfaire son travail, s'assoit sur eux. Le chanteur s'extrait du tas, à présent mi-homme, mi-danseuse avec la tête du comique !

Pourquoi ce film fait-il autant rire ? Parce que les actes et les gestes outranciers dépassent à un point inimaginable ce qu'il est permis de faire. Or, la transgression des limites propres à notre double burlesque vue dans les actes est ici redoublée par les réactions hyperboliques gestuelles du personnage, suscitant les mouvements frénétiques du corps burlesque et les exploits du corps morcelé. Comme dans les Boireau et les Onésime, il y a une exhibition de I'inconscient corporel absolument réjouissante et terrifiante à la fois. La grossièreté et d'autres comportements instinctuels vont de pair avec la domination des facultés inférieures, mais ici les films exhument quelque chose de plus profond que des actes : une strate inconsciente qui se fait voir à travers le corps. II y a une différence supplémentaire d'avec les burlesques américains des années vingt : les spectateurs français d'avant la Grande Guerre voyaient dans les bandes burlesques de leur époque les "symptômes » des pathologies nerveuses.

L'agitation corporelle et la gestuelle convulsive sont des émanations des facultés inférieures la domination du corps sur la raison, la perte de contrôle. Le lien avec la dégénérescence, la régression et la sauvagerie était aisé à établir pour les spectateurs autour de 1900, préoccupés par ces menaces envers la civilisation moderne. Malgré cette inquiétude, le corps de ce public fasciné mimait à son insu ces gestes burlesques outrés et le rire évacuait pour un moment la reconnaissance de ce Double à l'écran. 